\title{
Deformity Correction by Femoral Supracondylar Dome Osteotomy with Retrograde Intramedullary Nailing in Varus Deformity of the Distal Femur after Pathologic Fracture of Giant Cell Tumor
}

\author{
Moon-Jib Yoo, MD, Jae-Sung Yoo, MD, Jun-Yeul Lee, MD, and Chang-Hwan Hwang, MD \\ Department of Othopedic Surgery, Dankook University College of Medicine, Cheonan, Korea
}

\begin{abstract}
Angular deformities of the distal femur occur in congenital diseases or due to acquired causes, such as malunion after a fracture of the distal femur. Angular deformities of the lower extremities affect the mechanical axis, causing changes in the weight pressure on the articular surface. As a result, angular deformities quicken the progression of osteoarthritis. Therefore, correction of deformities should be performed to prevent the progression of osteoarthritis. Distal femoral osteotomy is one of the methods to correct angular deformities in unicompartmental osteoarthritis. However, femoral supracondylar dome osteotomy with retrograde intramedullary nailing in the distal femur with a varus deformity has been rarely reported. Herein, we describe a technique for femoral supracondylar dome osteotomy with retrograde intramedullary nailing in a varus deformity after a pathologic fracture of giant cell tumor in the distal femur with a review of the relevant literature.
\end{abstract}

Keywords: Femur, Deformity, Osteotomy, Intramedullary nailing

Angular deformities of the distal femur can accelerate progression of arthritis due to abnormal stress distribution across the joint caused by mechanical axis deviation ${ }^{1}$. To halt the progression of arthritis ${ }^{2}$, deformity correction techniques can be used, and femoral osteotomy is one of such techniques for unicompartmental arthritis of the knee with a severe angular deformity of the distal femur ${ }^{3}$.

Open wedge osteotomy, closed wedge osteotomy, and dome osteotomy are the currently available deformity correction techniques, and metal plating, intramedullary nailing, and ex-

Received July 23, 2013; Revised (1st) August 21, 2013; (2nd) September 24, 2013; (3rd) October 16, 2013; Accepted October 16, 2013

Correspondence to: Jae-Sung Yoo, MD

Department of Orthopaedic Surgery, Dankook University College of Medicine, 119 Dandae-ro, Dongnam-gu, Cheonan 330-997, Korea

Tel: +82-41-550-6579, Fax: +82-41-556-3238

E-mail: jsyoo1025@gmail.com

This is an Open Access article distributed under the terms of the Creative Commons Attribution Non-Commercial License (http://creativecommons.org/licenses/by-nc/3.0/) which permits unrestricted non-commercial use, distribution, and reproduction in any medium, provided the original work is properly cited. tramedullary nailing have been employed for fixation ${ }^{4)}$. Varus deformities of the distal femur after pathologic fractures of giant cell tumors have been rarely reported and femoral supracondylar dome osteotomy with retrograde intramedullary nailing has been performed in rare cases ${ }^{5}$. Here, we report the results of femoral supracondylar dome osteotomy with retrograde intramedullary nailing that was performed on a knee with a varus deformity secondary to pathologic fracture of giant cell tumor in the distal femur with a review of the relevant literature.

\section{Case Report}

A 46-year-old female patient visited our clinic with a complaint of persistent pain in the left knee that had lasted for one year. She had no history of trauma or infection. Physical examination revealed mild tenderness along the medial joint line and restricted range of motion in the affected knee (0-120 i) compared to the intact knee (0-140 pa). Although varus deformity of the knee was visible to the naked eye, joint instability was not present. Plain radiographs showed a varus deformity caused by malunion in the left distal femur, medial femorotibial joint space narrow- 
ing, and subchondral sclerosis (Fig. 1A). The umbilicus malleolar distance (UMD) was $84.8 \mathrm{~cm}$ on the intact side and $83.2 \mathrm{~cm}$ on the affected side. The spina malleolar distance was $80.2 \mathrm{~cm}$ on the intact side and $79.1 \mathrm{~cm}$ on the affected side. The patient did not complain of discomfort related to the leg length discrepancy. The femorotibial angle was measured as $21.5^{\circ}$ of varus, the mechanical lateral distal femoral angle (M-LDFA) was $104.3^{\circ}$ and the anatomical lateral distal femoral angle (A-LDFA) was $109.5^{\circ}$ in the left knee (Fig. 1B). Her medical history showed that she had been diagnosed with giant cell tumor of the distal femur ten years ago, which was treated with curettage and cryotherapy with liquid nitrogen and allograft (Fig. 2), and then the same treatment was performed 9 and 8 years ago, respectively, due to recurrence. Thereafter, she sustained a pathologic fracture in the same region after a slip and fall, which was treated conservatively (Fig. 3).

Deformity correction was determined necessary considering
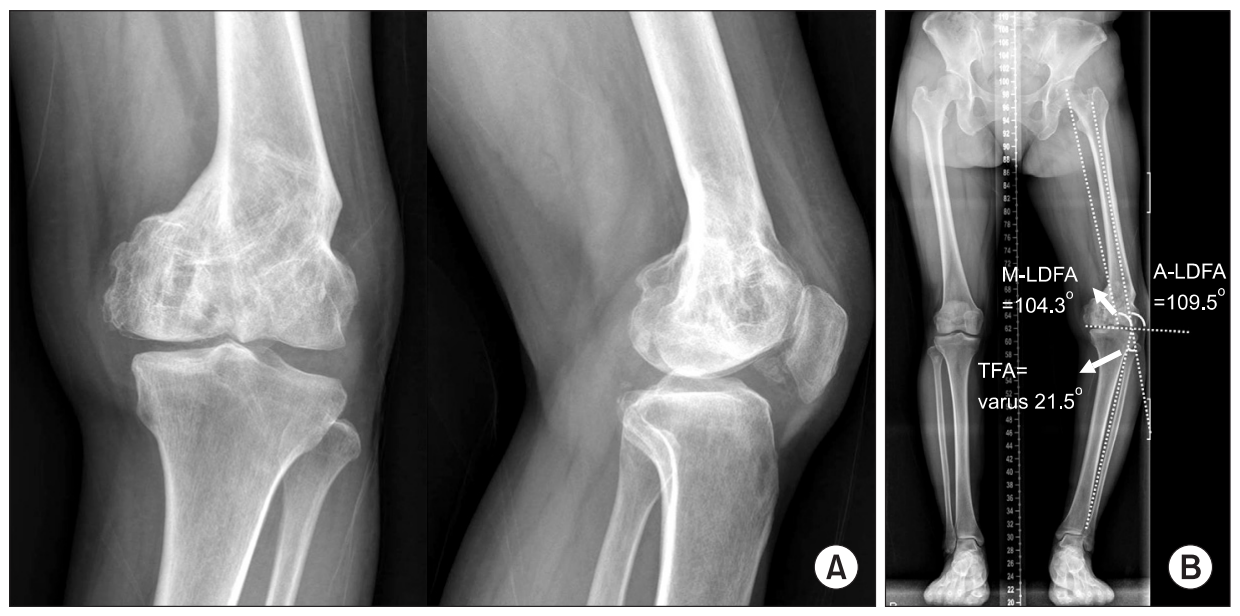

Fig. 1. Preoperative simple radiographs of the left knee showing (A) arthritic changes of the medial compartment with a varus deformity. (B) Long-leg weight bearing radiograph showing mechanical axis passing medial to the medial compartment of the knee and angular measurements of the knee. M-LDFA: mechanical lateral distal femoral angle, A-LDFA: anatomical lateral distal femoral angle, TFA: tibiofemoral angle.
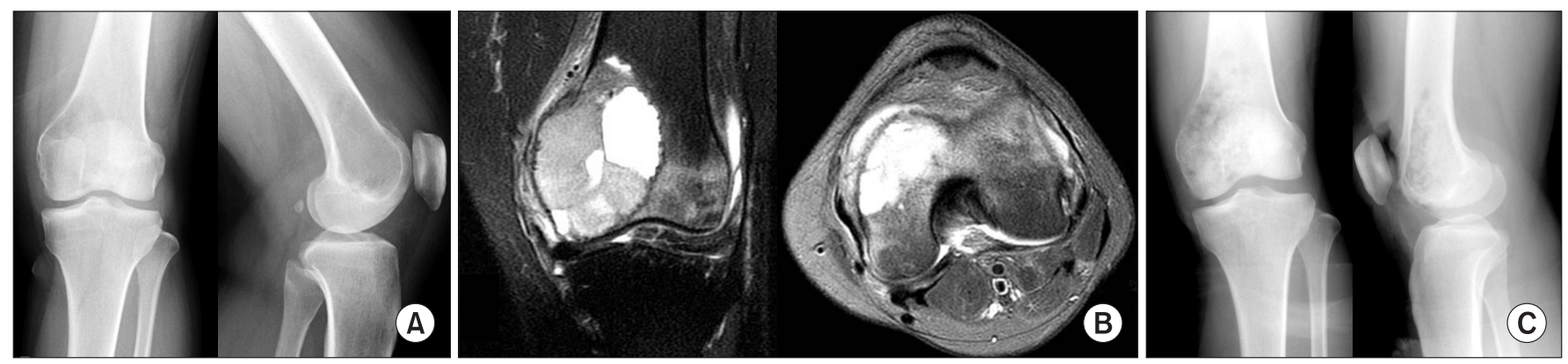

Fig. 2. (A) Radiographs of the left knee obtained when she was initially diagnosed with a giant cell tumor show a radiolucent mass lesion and focal cortex destruction at the anterior cortex of the medial femoral condyle. (B) T2 weighted images show the mass has an intermediate to high signal intensity. (C) Plain radiographs of the left knee obtained after curettage and allobone grafting.
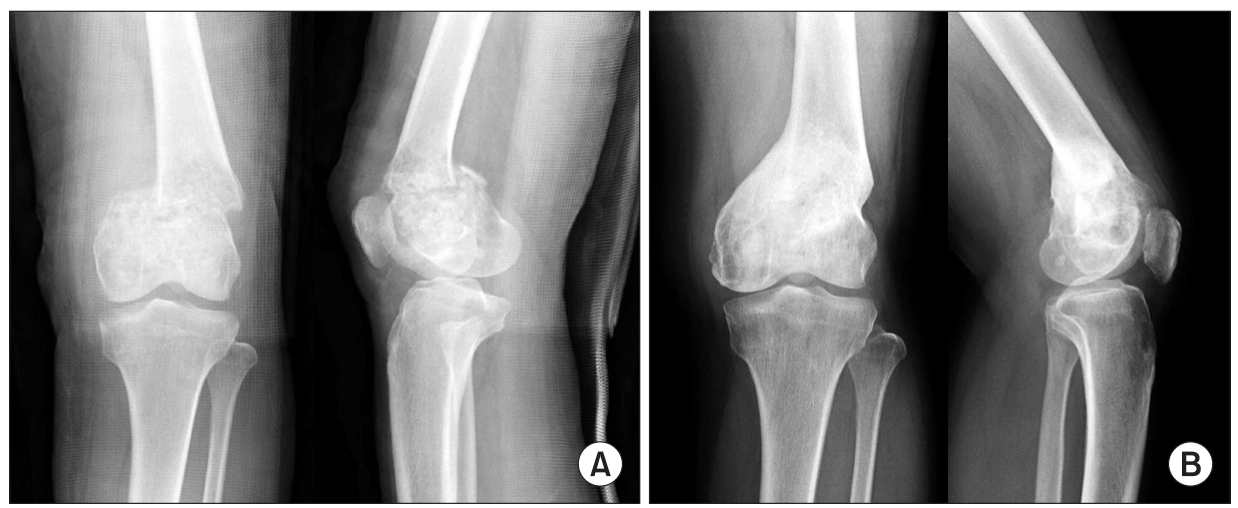

Fig. 3. (A) Plain radiographs obtained when she was diagnosed with a pathologic supracondylar fracture of giant cell tumor in the distal femur. (B) Radiographs obtained 6 months after injury shows malunion at the fracture site. 
the patient's young age and radiographic findings of severe varus deformity combined with arthritis in the medial compartment. Although the UMD on the affected knee was shorter than that on the intact knee $(83.2 \mathrm{~cm}$ vs. $84.8 \mathrm{~cm})$, there was no significant side-to-side difference in the total anatomical length of the femur and the tibia $(68.3 \mathrm{~cm}$ in the intact knee and $67.9 \mathrm{~cm}$ in the affected knee) and the patient did not complain of any discomfort. Thus, leg-length equalization was considered unnecessary. The correction angle was determined as $30^{\circ}$ with the aim of achieving $5^{\circ}-10^{\circ}$ varus of the feromotibial angle postoperatively. It was estimated that the 30 correction for the femur with a diameter of 4 $\mathrm{cm}$ would result in $2.3 \mathrm{~cm}$ of leg lengthening with use of a medial open technique whereas $2.3 \mathrm{~cm}$ of leg shortening with use of a
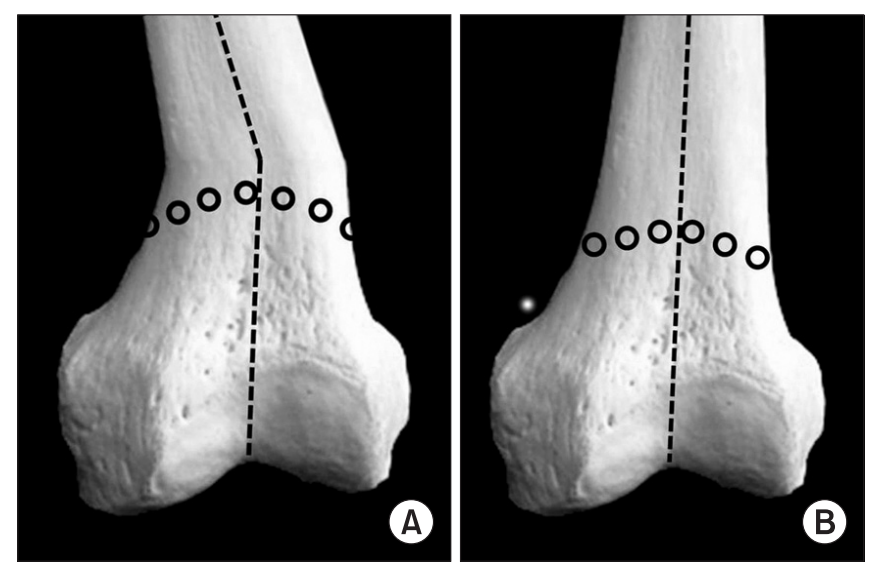

Fig. 4. (A) Supracondylar of the distal femur was cut in a dome shape. Predrilling was helpful for localizing the supracondylar dome osteotomy site. (B) After the supracondylar dome osteotomy, angular correction was possible without changing the leg length.

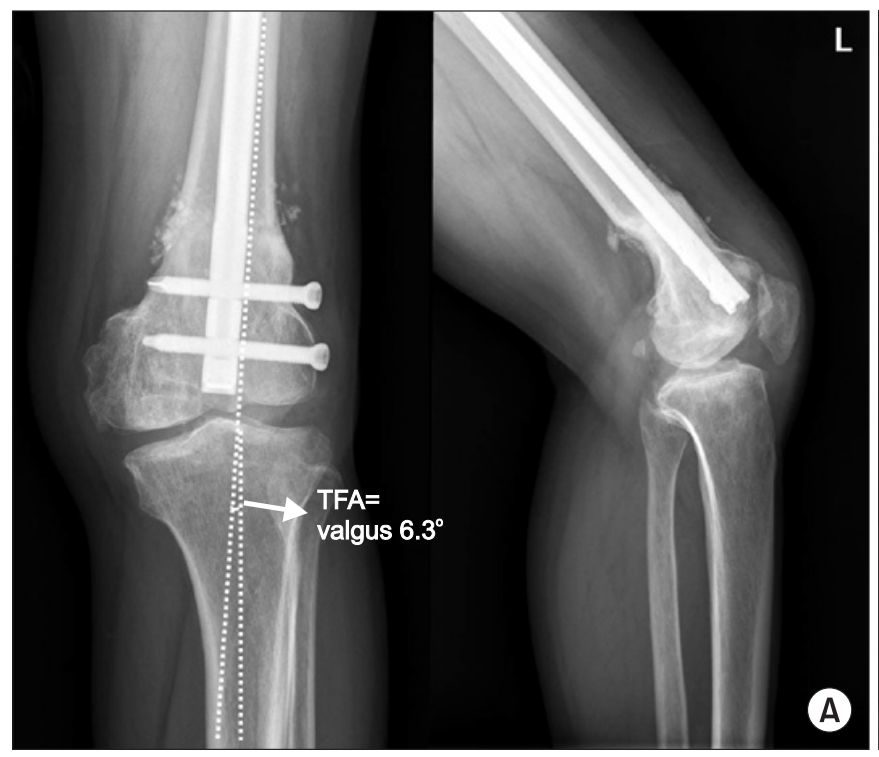

lateral closed technique. Thus, we determined to perform a corrective dome osteotomy that would have no significant influence on the leg length.

Adhesiolysis around the anterior joint capsule and quadriceps lengthening were considered necessary due to limited flexion. For the osteotomy, we determined to use an anteromedial approach as in the previous surgery. After making a $15 \mathrm{~cm}$ skin incision on the anteromedial aspect, adhesiolysis around the anterior joint capsule and quadriceps lengthening using Z-plasty were performed. After verifying the recovery of complete range of motion, the rectus femoris and patellar bone were retracted laterally to expose the distal femur. Evidence of arthritis was observed across the medial articular surface of the distal femur, but specific findings that suggest a giant cell tumor were not noted. The center of rotation angulation (CORA) was located under fluoroscopy. After determining the dome osteotomy site from the femoral supracondylar with an interval of $10.0 \mathrm{~mm}$ using a $2.0 \mathrm{~mm}$ drill bit, the osteotomy was performed using a parallel saw blade (Synthes GmbH, Solothurn, Switzerland) (Fig. 4). The varus deformity was corrected and the mechanical and anatomical axes were assessed under the guidance of fluoroscopy. Without an additional incision, retrograde intramedullary nailing was performed through the distal femur for fixation. PolyBone (Kyungwon Medical Co., Seoul, Korea) containing polyphosphonate and bone morphogenetic protein was additionally inserted (Fig. 5). For six postoperative weeks, long leg cast immobilization was applied, and active joint exercises and partial weight-bearing were allowed. Full weight-bearing was permitted from the 12th postoperative week. At 24 weeks postoperatively, bone union and
Fig. 5. Plain radiography after 6 months later deformity correction by supracondylar dome osteotomy with retrograde intramedullary nailing. (A, B) Knee and whole lower extremity plain radiography shows correction of deformity and union of osteotomy site. TFA: tibiofemoral angle, M-LDFA: mechanical lateral distal femoral angle, A-LDFA: anatomical lateral distal femoral angle. 
normal range of motion were achieved. The femorotibial angle was $6.3^{\circ}$ of varus, the M-LDFA was 78.6, and the anatomical ALDFA was 76.3 at 24 weeks postoperatively (Fig. 5).

\section{Discussion}

Mirels ${ }^{6}$ suggested that the possibility of sustaining a pathologic fracture should be assessed based on the size, location, pattern, and pain level of a tumor, and preventive fixation should be performed, if necessary, to lower the risk of pathologic fractures and produce effective treatment outcomes. In the study, preventive fixation was recommended for lesions with scores of 9 or higher according to the Mirels scoring system (Table 1). At the initial assessment, our patient presented with an Enneking stage 3 lesion accompanied with a cortical defect, and it was retrospectively classified as a score 9 lesion according to the Mirels scoring system. We think that the complications of pathologic fracture that occurred after two instances of relapse could have been averted with preventive fixation if the impending risk of fracture could have been estimated through thorough assessment.

Angular deformities affect the mechanical axis of the lower limb and thus alter stress distribution across the joint during standing and walking, which may facilitate the progression of arthritis. Accordingly, in the presence of early arthritis in the medial or lateral compartment, deformity correction techniques can be useful for slowing the progression arthritis ${ }^{3)}$. Depending on the extent and site of an angular deformity, proximal tibial osteotomy or distal femoral osteotomy can be performed ${ }^{7,8}$.

Distal femoral osteotomy can be done in an open or closed manner. Open osteotomy is advantageous for obtaining leg lengthening, but bone grafting should be performed additionally and bone union is difficult to be achieved. On the other hand, closed osteotomy does not require bone grafting, but causes limb shortening ${ }^{4)}$. Supracondylar dome osteotomy is advantageous in various aspects: 1) it allows deformity correction without any leg length change, 2) it is performed in the femoral methaphysis that is primarily composed of cortical bone, and thus facilitates bone union through the wide contact surfaces at the osteotomy site and, 3) it allows for simultaneous correction of varus/valgus and

Table 1. Mirels Scoring System

\begin{tabular}{ccccl}
\hline Score & Site of lesion & Size of lesion & Nature of lesion & \multicolumn{1}{c}{ Pain } \\
\hline 1 & Upper limb & $<1.3$ & Blastic & Mild \\
2 & Lower limb & $1.3-2.3$ & Mixed & Moderate \\
3 & Trochanteric region & $>2.3$ & Lytic & Functional \\
\hline
\end{tabular}

flexion/extension deformities through the dome shaped resected surface ${ }^{8)}$.

Determination on the method of fixation that follows femoral osteotomy should be based on the accuracy of reduction, operative time, range of motion, and understanding on the complications inherent to the operative technique ${ }^{4}$. Metal plate fixation is effective for obtaining early rigid fixation, but the downsides include extensive soft tissue damage, difficulty of residual correction after surgery, and metal failure. External fixation allows for gradual correction of the remaining deformity after surgery, but pin site infection, restriction on early range of motion exercises, and device-related discomfort are unavoidable ${ }^{4}$. Intramedullary fixation is advantageous for avoiding soft tissue injury compared to the metal plate fixation, but it is not conducive to obtain rigid fixation in cases of metaphyseal osteotomy ${ }^{5,9}$. However, Wich et al. ${ }^{5)}$ reported satisfactory results of distal femoral osteotomy with internal fixation using a retrograde nail. In addition, consideinrg that femoral supracondylar dome osteotomy can be performed via an anterior approach ${ }^{8)}$, retrograde intramedullary nailing can be a useful fixation method that can be carried out without an additional incision on the medial or lateral side.

In our patient, soft tissue release and quadriceps lengthening were performed via an anteromedial approach that had been utilized in the previous treatment. Thus, intramedullary retrograde nailing was chosen for fixation to obviate soft tissue damage caused by an additional incision. Complete bone union and recovery of range of motion were obtained at 6 months after surgery.

\section{Conclusions}

Supracondylar dome osteotomy with intramedullary retrograde nailing can be effective for the treatment of angular deformities of the distal femur.

\section{Conflict of Interest}

No potential conflict of interest relevant to this article was reported.

\section{References}

1. Tetsworth K, Paley D. Malalignment and degenerative arthropathy. Orthop Clin North Am. 1994;25:367-77.

2. Martinez de Albornoz P, Leyes M, Forriol F, Del Buono A, Maffulli N. Opening wedge high tibial osteotomy: plate 
position and biomechanics of the medial tibial plateau. Knee Surg Sports Traumatol Arthrosc. 2013 Apr 30. [Epub]. http://dx.doi.org/10.1007/s00167-013-2517-9.

3. Kaissi AA, Farr S, Ganger R, Hofstaetter JG, Klaushofer K, Grill F. Treatment of varus deformities of the lower limbs in patients with achondroplasia and hypochondroplasia. Open Orthop J. 2013;7:33-9.

4. Seah KT, Shafi R, Fragomen AT, Rozbruch SR. Distal femoral osteotomy: is internal fixation better than external? Clin Orthop Relat Res. 2011;469:2003-11.

5. Wich M, Veltin J, Hollen I, Letsch R. Multidimensional corrective osteotomy of the distal femur using a retrograde femoral nail. Unfallchirurg. 1999;102:652-5.

6. Mirels H. Metastatic disease in long bones: a proposed scoring system for diagnosing impending pathologic fractures.
Clin Orthop Relat Res. 1989;(249):256-64.

7. Sharma L, Eckstein F, Song J, Guermazi A, Prasad P, Kapoor D, Cahue S, Marshall M, Hudelmaier M, Dunlop D. Relationship of meniscal damage, meniscal extrusion, malalignment, and joint laxity to subsequent cartilage loss in osteoarthritic knees. Arthritis Rheum. 2008;58:1716-26.

8. Luna-Pizarro D, Moreno-Delgado F, De la Fuente-Zuno JC, Meraz-Lares G. Distal femoral dome varus osteotomy: surgical technique with minimal dissection and external fixation. Knee. 2012;19:99-102.

9. Kettunen J, Makela A, Miettinen H, Nevalainen T, Heikkila M, Tormala P, Rokkanen P. Fixation of distal femoral osteotomy with an intramedullary rod: early failure of carbon fibre composite implant in rabbits. J Biomater Sci Polym Ed. 1999;10:715-28. 